\title{
Molecular Heat Engines: Quantum Coherence Effects
}

\author{
Feng Chen ${ }^{1}$, Yi Gao ${ }^{2,+}$ and Michael Galperin ${ }^{2, *}$ (1) \\ 1 Department of Physics, University of California San Diego, La Jolla, CA 92093, USA; fec011@ucsd.edu \\ 2 Department of Chemistry and Biochemistry, University of California San Diego, La Jolla, CA 92093, USA; \\ yigao1983@gmail.com \\ * Correspondence: migalperin@ucsd.edu; Tel.: +1-858-246-0511 \\ $\dagger$ Current address: PIMCO, Investment Management, Newport Beach, CA 92660, USA.
}

Received: 29 July 2017; Accepted: 2 September 2017; Published: 4 September 2017

\begin{abstract}
Recent developments in nanoscale experimental techniques made it possible to utilize single molecule junctions as devices for electronics and energy transfer with quantum coherence playing an important role in their thermoelectric characteristics. Theoretical studies on the efficiency of nanoscale devices usually employ rate (Pauli) equations, which do not account for quantum coherence. Therefore, the question whether quantum coherence could improve the efficiency of a molecular device cannot be fully addressed within such considerations. Here, we employ a nonequilibrium Green function approach to study the effects of quantum coherence and dephasing on the thermoelectric performance of molecular heat engines. Within a generic bichromophoric donor-bridge-acceptor junction model, we show that quantum coherence may increase efficiency compared to quasi-classical (rate equation) predictions and that pure dephasing and dissipation destroy this effect.
\end{abstract}

Keywords: efficiency; quantum coherence; Green functions; molecular electronics

\section{Introduction}

Single molecules are used as building blocks of molecular devices for electronics, biosensors, nanoscale motors, controllable chemical reactivity and energy transfer [1-3]. The development of nano-fabrication led to tremendous progress in the ability to detect and manipulate molecules on surfaces and in junctions [4-9]. Due to the small size of nanodevices, their characterization is necessarily quantum, and interference is expected to be crucial in the response of molecular electronic devices. Experimental measurements have demonstrated quantum coherence effects on the transport [10-12] and optical response $[13,14]$ of molecular junctions. The importance of quantum coherence in energy transfer was demonstrated in experimental studies of the initial stages of photosynthesis [15-18].

Understanding and controlling the combined motion of charges and excitations (energy) is crucial for the development of new materials and state-of-the-art guiding principles for building efficient energy conversion and storage devices. Historically, this research has been focused on thermoelectric properties in bulk materials [19]. With the development of nanofabrication techniques, the study of thermoelectric properties at the nanoscale attracted a lot of attention experimentally [20-28] and theoretically [29-40]. The small size of these nanodevices gives rise to new physical phenomena (such as quantum coherence) that are not present at the macroscopic level, and which promise to improve the performance of energy conversion. These studies are concerned with characterizing charge and energy fluxes in the system.

A closely related set of works utilized the thermodynamic approach to determine the efficiency of photoelectric devices [41-45]. With rate (Pauli) equations employed in the thermodynamic description of such devices, quantum coherence could not be fully taken into account (see below). The effect of coherence on the thermodynamics of quantum heat engines consisting of n-level systems coupled 
to thermal bath(s) was considered in a number of publications [46-50] at the Lindblad-Redfield level of theory. These considerations were either restricted to closed systems or disregarded charge transfer between the system and the baths (the latter is inherent in molecular devices). Thus, naturally also in thermodynamic studies, the question of how quantum coherence affects a molecular device performance can be raised.

We note in passing that the distinction between populations and coherences (diagonal and off-diagonal elements of the density matrix) is basis dependent. For example, transforming the local basis to the eigenbasis of the system accounts for coherences of the local basis simply by converting them into populations in the eigenbasis. For an isolated system, local basis coherences are taken into account exactly as a result of such a transformation. In the presence of baths, for the consideration to be complete one has to account also for bath-induced coherences between the eigenstates of the system. The latter cannot be treated properly at the Lindblad-Redfield quantum master equation (QME) level of theory (one has to go to at least the fourth order in system-bath coupling to account for the coherences [51]), while approximate consideration may lead to qualitative failures (see, e.g., [52-54]). In addition, Lindblad-Redfield QME does not allow to model the gradual transition from coherent to incoherent transport. The latter is the focus of our study.

Here, we consider a generic donor-bridge-acceptor (DBA) molecular system, which is coupled to fermionic baths and is driven against the bias applied by solar photons. This setup is a simple model for a continuous steady-state heat engine [55-57], whose thermoelectric efficiency was previously considered in $[41,42,44]$ with the effects of quantum coherence disregarded. The latter were shown to play an important role in the charge and energy transport in similar models of DBA molecular junctions [58,59].

We utilize nonequilibirum Green function (NEGF) methodology [60], which is capable of accounting for quantum coherence in an open nonequilibrium system, to study the effects of quantum coherence on the average efficiency of photoelectric molecular devices. For simplicity, our consideration is restricted to a non-interacting (electron-electron and electron-vibration interactions are disregarded) molecular system, although intra-system interactions in principle can be taken into account within many-body flavors of the methodology [61-65]. We show that quantum coherence may lead to an increase and a decrease in the efficiency of the device and study the transition to a quasi-classical regime by destroying coherence with pure dephasing or dissipation. The former is achieved by employing a Büttiker probe [66], and the latter is induced by increasing the strength of the system-contacts coupling. We note in passing that although a general formulation of quantum thermodynamics for current-carrying junctions has not yet been established $[67,68]$, the formulation is clear as long as the junction operates in a steady-state [69,70]. The latter is the situation considered here.

The paper is structured as follows: In Section 2, we introduce the model and discuss the technical details of the simulations. We present the results of the numerical simulations and compare them with previously published (coherence-free) studies in Section 3. We draw conclusions in Section 4.

\section{Model}

We consider a molecular junction comprised of a DBA molecular complex coupled to metallic contacts $L$ and $R$ (see Figure 1). The contacts are equilibrium reservoirs of free charge carriers maintained at the same temperature $T$. The junction is biased so that the electrochemical potential of contact $L$ is lower than that of $R, \mu_{L}<\mu_{R}$. The donor and the acceptor are modeled as two-level (highest occupied molecular orbital-lowest unoccupied molecular orbital, HOMO-LUMO) systems. Following [44], we assume that the HOMO of the acceptor is always populated (i.e., does not participate in transport), and thus can be disregarded. The bridge provides super-exchange coupling between the donor and the acceptor and is accounted for by effective electron hopping matrix element $t$. The donor is subjected to solar radiation which is modeled by coupling to a thermal bath $S$ of high (solar) temperature $T_{S}\left(T_{S} \gg T\right)$. Electron transfer is allowed between the LUMOs of the donor and the acceptor; thus, solar radiation drives the electronic flux against the applied bias (heat engine). 
Bridge-induced coupling between the LUMOs (levels 2 and 3 in Figure 1) is the cause of intra-molecular quantum coherence. For an isolated two-level system, such coherence leads to permanent Rabi oscillations in the electron population on both levels. In our model, these oscillations are damped by coupling to baths (solar radiation and contacts) with the damping rate depending on the strength of the couplings.

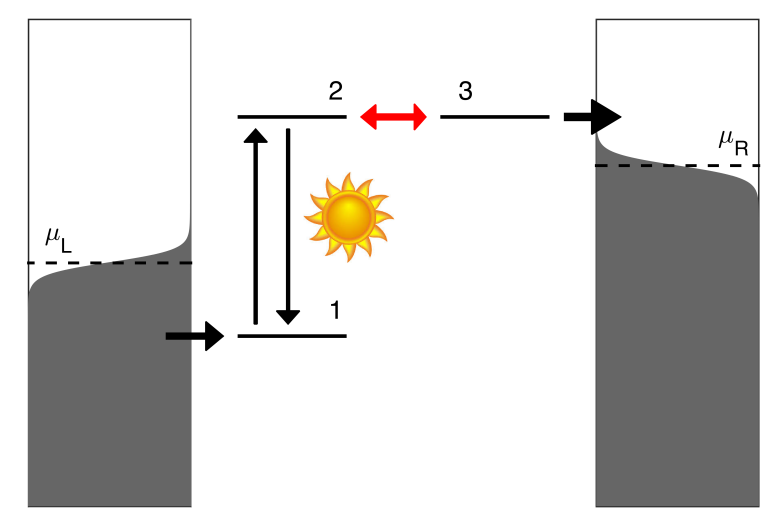

Figure 1. Sketch of the molecular donor-bridge-acceptor (DBA) heat engine model.

The Hamiltonian of the model is

$$
\hat{H}=\hat{H}_{M}+\sum_{B=L, R, S}\left(\hat{H}_{B}+\hat{V}_{B}\right),
$$

where the $\hat{H}_{M}$ and $\hat{H}_{B}$ Hamiltonians represent the molecule $(M)$, contacts ( $L$ and $R$ ) and thermal bath $(S)$, respectively. $\hat{V}_{B}$ couples the molecule to the baths (contacts and Sun radiation). Explicit expressions are

$$
\begin{aligned}
& \hat{H}_{M}=\sum_{m=1}^{3} \varepsilon_{m} \hat{d}_{m}^{\dagger} \hat{d}_{m}+t\left(\hat{d}_{2}^{\dagger} \hat{d}_{3}+\hat{d}_{3}^{+} \hat{d}_{2}\right) \\
& \hat{H}_{L(R)}=\sum_{k \in L(R)} \varepsilon_{k} \hat{c}_{k}^{\dagger} \hat{c}_{k} ; \quad \hat{H}_{S}=\sum_{\alpha \in S} \omega_{\alpha} \hat{a}_{\alpha}^{\dagger} \hat{a}_{\alpha} \\
& \hat{V}_{L}=\sum_{\ell \in L}\left(V_{\ell} \hat{d}_{1}^{\dagger} \hat{c}_{\ell}+H . c .\right) ; \quad \hat{V}_{R}=\sum_{r \in R}\left(V_{r} \hat{d}_{3}^{\dagger} \hat{c}_{r}+H . c .\right) \\
& \hat{V}_{S}=\sum_{\alpha \in S}\left(U_{\alpha} \hat{D}^{\dagger} \hat{a}_{\alpha}+H . c .\right) .
\end{aligned}
$$

Here, $\hat{d}_{m}^{\dagger}\left(\hat{d}_{m}\right)$ and $\hat{c}_{k}^{\dagger}\left(\hat{c}_{k}\right)$ create (annihilate) the electron at the molecular level $m$ or contact state $k$, respectively. $\hat{D}^{+}=\hat{d}_{2}^{+} \hat{d}_{1}$ is the donor excitation operator. $\hat{a}_{\alpha}^{+}\left(\hat{a}_{\alpha}\right)$ creates (annihilates) excitation quanta in the thermal bath. We previously discussed a similar model $[58,59]$ in a study of the quantum coherence effects on electron transport in DBA junctions. Einax et al. [42,44] utilized the model in consideration of a molecular heat engine within a hopping transport regime. Here, we elucidate the effects of intra-molecular quantum coherence (Rabi oscillation between the LUMOs, levels 2 and 3; see Figure 1) on the thermodynamic performance of the engine.

The response properties of the junction can be conveniently expressed in terms of NEGFs. In particular, we use single- and two-particle electron Green functions defined on the Keldysh contour as (here and below $e=\hbar=k_{B}=1$ )

$$
G_{m_{1} m_{2}}\left(\tau_{1}, \tau_{2}\right)=-i\left\langle\mathcal{T}_{c} \hat{d}_{m_{1}}\left(\tau_{1}\right) \hat{d}_{m_{2}}^{\dagger}\left(\tau_{2}\right)\right\rangle ; \quad \mathcal{G}\left(\tau_{1}, \tau_{2}\right)=-i\left\langle\mathcal{T}_{c} \hat{D}\left(\tau_{1}\right) \hat{D}^{\dagger}\left(\tau_{2}\right)\right\rangle
$$


Here, $m_{1}$ and $m_{2}$ indicate the molecular levels, $\mathcal{T}_{c}$ is the contour ordering operator and $\tau_{1}$ and $\tau_{2}$ are the contour variables. The single-particle Green function is obtained by solving the Dyson equation

$$
G_{m_{1} m_{2}}\left(\tau_{1}, \tau_{2}\right)=G_{m_{1} m_{2}}^{(0)}\left(\tau_{1}, \tau_{2}\right)+\sum_{m_{3}, m_{4}} \int_{\mathcal{c}} d \tau_{3} \int_{\mathcal{c}} d \tau_{4} G_{m_{1} m_{3}}^{(0)}\left(\tau_{1}, \tau_{3}\right) \Sigma_{m_{3} m_{4}}\left(\tau_{3}, \tau_{4}\right) G_{m_{4} m_{2}}\left(\tau_{4}, \tau_{2}\right),
$$

where $G_{m_{1} m_{2}}^{(0)}\left(\tau_{1}, \tau_{2}\right)$ is the single-particle Green function in the absence of coupling to baths and

$$
\Sigma_{m_{1} m_{2}}\left(\tau_{1}, \tau_{2}\right)=\sum_{B=L, R, S} \Sigma_{m_{1} m_{2}}^{B}\left(\tau_{1}, \tau_{2}\right)
$$

is the total electron self-energy due to coupling to the baths. In our consideration, we use second-order (in system-baths couplings) diagrammatic expansion. The procedure is self-consistent, because single-electron self-energy due to coupling to the radiation field $\Sigma_{m_{1} m_{2}}^{S}\left(\tau_{1}, \tau_{2}\right)$ depends on the single-electron Green function $G_{m_{1} m_{2}}\left(\tau_{1}, \tau_{2}\right)$, while the latter is defined by the self-energy. Below, we utilize level populations,

$$
n_{m}=-i G_{m m}^{<}(t, t) \quad(m=1,2,3),
$$

at subsequent iteration steps to judge the convergence of the procedure. Here, $G^{<}$is a lesser projection of the single-particle Green function. Explicit expressions of the self-energies are given in Appendix A.

To evaluate the two-particle Green function $\mathcal{G}$, we employ an approximation

$$
\mathcal{G}\left(\tau_{1}, \tau_{2}\right) \approx-i G_{11}\left(\tau_{2}, \tau_{1}\right) G_{22}\left(\tau_{1}, \tau_{2}\right)
$$

which disregards multi-photon processes in the evaluation of the heat flux (see below). This approximation was employed in earlier studies [71], and for the parameters of the simulations (strength of coupling to the radiation field), the approximation is reasonable.

Below, we calculate the particle flux at the interface with the right contact, $I_{R}$, and the energy (heat) flux at the interface with the solar bath $S, J_{S}$. In terms of these fluxes, the average thermodynamic efficiency of the molecular heat engine is defined as

$$
\eta=\frac{\left(\mu_{R}-\mu_{L}\right) I_{R}}{J_{S}} \equiv \frac{P}{J_{S}}
$$

where $P \equiv\left(\mu_{R}-\mu_{L}\right) I_{R}$ is the power of the engine. We are interested in the efficiency at the maximum power $\eta_{\max }$. Within NEGF, fluxes $I_{R}$ and $J_{S}$ are defined as the rates of the change in the electronic population in $R$ and the energy in $S$, respectively:

$$
I_{R}=-\frac{d}{d t} \sum_{r \in R}\left\langle\hat{c}_{r}^{\dagger}(t) \hat{c}_{r}(t)\right\rangle ; \quad J_{S}=-\frac{d}{d t} \sum_{\alpha \in S} \omega_{\alpha}\left\langle\hat{a}_{\alpha}^{\dagger}(t) \hat{a}_{\alpha}(t) .\right\rangle
$$

They can be expressed exactly in terms of the Green functions and self-energies. At steady state within the NEGF, fluxes $I_{R}$ and $J_{S}$ are (see Appendix B for the derivation)

$$
\begin{gathered}
I_{R}=\operatorname{Tr} \int_{-\infty}^{+\infty} \frac{d E}{2 \pi}\left(\Sigma^{R<}(E) G^{>}(E)-\Sigma^{R>}(E) G^{<}(E)\right), \\
J_{S}=-\int_{0}^{\infty} \frac{d \omega}{2 \pi} \omega\left(\Pi^{<}(\omega) \mathcal{G}^{>}(\omega)-\Pi^{>}(\omega) \mathcal{G}^{<}(\omega)\right) .
\end{gathered}
$$

Here, $\operatorname{Tr}[\ldots]$ is the trace over the molecular levels, $\Sigma^{R<}<(E)$ and $\Sigma^{R>}(E)$ are defined in Equations (A4) and (A5), $\Pi^{<}(\omega)$ and $\Pi^{>}(\omega)$ are given in Equations (A11) and (A12). 


\section{Results}

We present the results of the simulations for the DBA heat engine model (see Figure 1). Unless stated otherwise, the parameters of the simulation are ambient temperature $T=300 \mathrm{~K}$ (this is the temperature of the contacts $L$ and $R$ ), temperature of the Sun $T_{S}=6000 \mathrm{~K}$ (this is the temperature of the thermal bath $S$ ), molecular levels $\varepsilon_{1}=-0.5 \mathrm{eV}$ and $\varepsilon_{2}=\varepsilon_{3}=0.8 \mathrm{eV}$, donor-acceptor electron hopping $t=0.1 \mathrm{eV}$, contacts electron escape rates $\Gamma_{L}=\Gamma_{R}=0.01 \mathrm{eV}$ and the energy dissipation rate to the thermal bath $\gamma=0.01 \mathrm{eV}$. Fermi energy is taken as the origin, $E_{F}=0$, and the junction is biased in contact $R: \mu_{L}=E_{F}$ and $\mu_{R}=E_{F}+|e| V_{s d}$ (e is the electron charge). Here, $V_{s d}$ is the bias across the junction. Gate potential $V_{g}$ is applied to the acceptor; the position of the acceptor LUMO is $\varepsilon_{3}+|e| V_{g}$. Simulations are performed on the energy grid spanning the region from -3 to $3 \mathrm{eV}$ with step $5 \times 10^{-4} \mathrm{eV}$. Convergence is checked by comparing the level populations in the subsequent iterations of the self-consistent solution of the Dyson equation. The convergence tolerance is $10^{-5}$.

Figure 2 shows that quantum coherence between the LUMOs of the donor and acceptor results in the two-peak structure of the engine power dependence on the bias at the fixed gate potential (see the top panel). The effect can be easily understood if one transforms in the eigenbasis of the molecular system. In this basis, ground state $\left(\varepsilon_{1}\right)$ is coupled by Sun radiation to two excited eigenstates. Each of the excited states yields a separate channel for electron transfer into contact $R$. The strength of the excited states' coupling to the Sun radiation and the contact $R$ depends on gate potential $V_{g}$. Thus, the two-peak structure indicates the presence of two scattering eigenchannels with the dominant channel defined by detuning of the molecular system LUMOs (see Figure 2b). Note that this channel control is to some extent similar to consideration of [72] with the role of the resonant driving field there played by the gate potential in our consideration. Note also that channel control by level detuning does not affect the overall thermodynamic formulation, because (for static levels) no external thermodynamic forces are acting on the system.

While maximum power (see Figure 2c) is insensitive to the change in the dominant scattering channel, the thermodynamic efficiency at maximum power shows a non-monotonic behavior due to the sudden change in the bias at which the device performs at maximum power when the dominant channel is switched. We stress that this behavior is possible only due to the presence of quantum coherence in the molecular system and that when coherence is destroyed the efficiency at maximum power attains its monotonic dependence on level detuning in agreement with the classical study of the system [42].

To destroy system coherence, we employ Büttiker probes coupled to LUMOs of the donor and the acceptor. Büttiker probes are widely used in quantum transport literature. They are modeled as an additional bath (represented by additional self-energy), whose role is to induce dephasing in the system. The probes should destroy the phase at the same time not allowing for either electron or energy exchange between the system and the probe. Following [66], we introduce the probes by considering additional local self-energies

$$
\Sigma_{m m}\left(\tau_{1}, \tau_{2}\right)=\delta \cdot G_{m m}\left(\tau_{1}, \tau_{2}\right) \quad(m=2,3)
$$

Here, $\delta$ is the dephasing parameter. This self-energy can be derived considering the electron-phonon interaction in the second order of the diagrammatic expansion [60] in the limit of low phonon frequency [59]. Such physical insight shows that neither electron nor energy flux can be induced between the system and the probe. Thus, the probe can destroy only quantum coherence in the molecular system. 

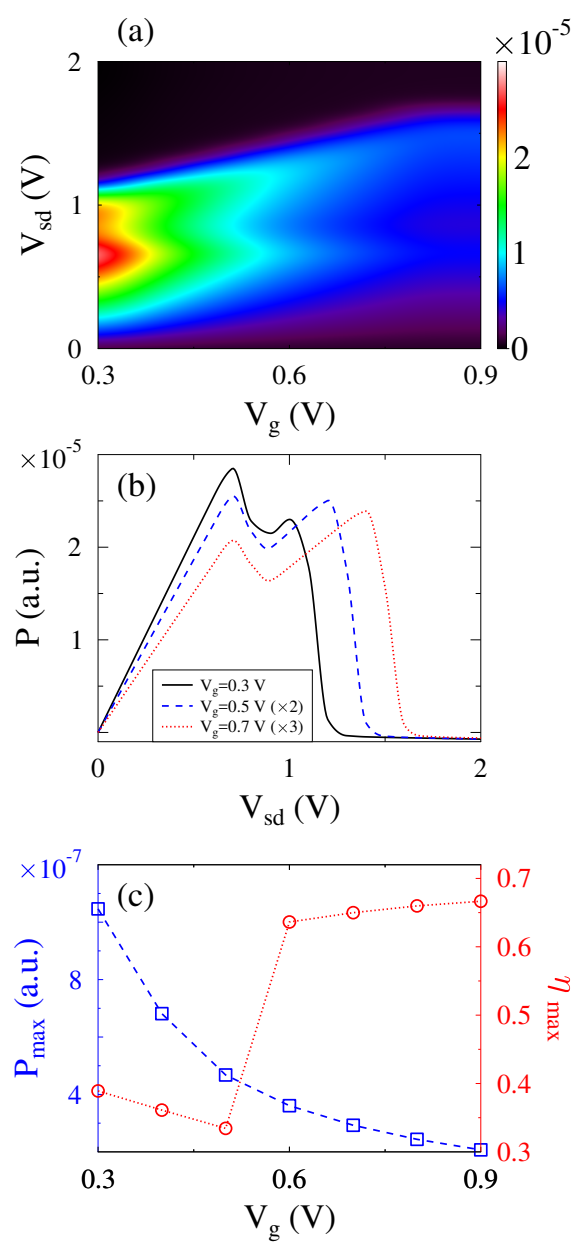

Figure 2. Effects of coherence in the DBA heat engine (Figure 1). Shown are (a) a map of the engine power $P$ vs. detuning of LUMOs, $V_{g}$, and bias across the junction, $V_{s d} ;(\mathbf{b})$ power $P$ vs. bias $V_{s d}$ for the three gate potentials (see inset; the results for $V_{g}=0.5$ and $0.7 \mathrm{~V}$ are scaled by a factor of 2 and 3 , respectively); (c) the dependence of the maximum power $P_{\max }$ (dashed line, squares) and the efficiency at maximum power $\eta_{\max }$ (dotted line, circles) on gate potential $V_{g}$.

Figure 3 demonstrates the effect of coherence destruction with Büttiker probes. The dephasing parameter employed in the simulation is $\delta=0.02 \mathrm{eV}^{2}$. Introducing the probe has two effects on the device's performance. First, by destroying coherence the two transport channel situation in the purely coherent case changes to a single transport channel situation in the purely classical (hopping) case. The dephasing parameter employed in the simulation partially destroys system coherence; therefore, the second channel is less prominent (compare panels (a) and (b) in Figures 2 and 3), although the impact of coherence on efficiency is still pronounced (compare the dotted lines in Figure 3c). Note that destroying coherence increases the efficiency for $V_{g}<0.6 \mathrm{~V}$, while decreasing it for $V_{g} \geq 0.6 \mathrm{~V}$. This indicates the prevalence (in the purely coherent case) of destructive and constructive interference in the two regions. Second, destroying coherence leads to a slight increase in the maximum power (compare the dashed lines in Figure 3c). The effect is due to the disruption by the Büttiker probe of the Rabi oscillations between the donor and acceptor LUMOs: The electron that previously spent time in the system now escapes faster in the right contact, which results in an increase in the current. 

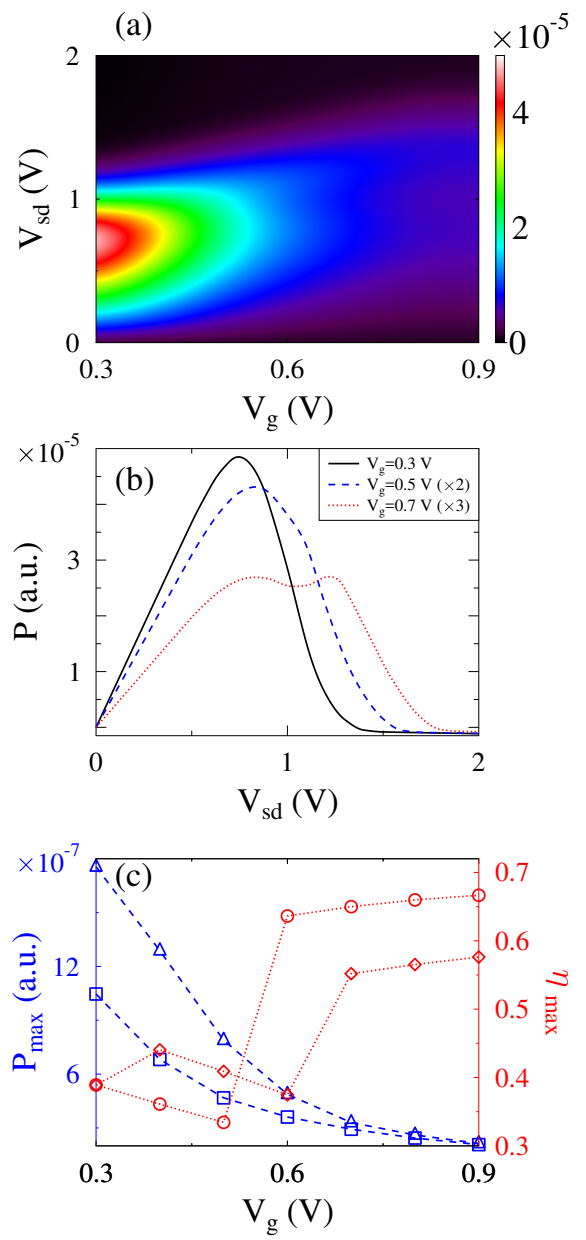

Figure 3. Coherence destruction on efficiency of the DBA heat engine by a Büttiker probe. Shown are (a) a map of the engine power $P$ vs. detuning of LUMOs, $V_{g}$, and bias across the junction, $V_{s d}$; (b) power $P$ vs. bias $V_{s d}$ for the three gate potentials (see inset; the results for $V_{g}=0.5$ and $0.7 \mathrm{~V}$ are scaled by a factor of 2 and 3, respectively); (c) the dependence of the maximum power $P_{\max }$ (dashed line, triangles) and efficiency at maximum power $\eta_{\max }$ (dotted line, diamonds) on gate potential $V_{g}$. The results presented in Figure 2c (coherent transport, $\delta=0$ )—squares (dashed line) and circles (dotted line)—are given for comparison.

Another way to destroy system coherence is by increasing dissipation due to coupling to contacts (increase escape rate parameters $\Gamma_{L}$ and $\Gamma_{R}$ ). We use $\Gamma_{L}=\Gamma_{R}=0.05 \mathrm{eV}$ in the simulations. Similar to the Bütiiker probe case, here the destruction of coherence results in a transition from two-channel to single-channel transport (and from quantum to classical behavior). The difference between the two cases is that while the former is pure decoherence, which results in a transition from coherent transport to hopping, the latter effectively eliminates the acceptor LUMO by incorporating it into the right contact. Thus, the extreme of the Bütiiker probe is the classical model of [42] while strong acceptor-contact coupling results in the classical consideration of [41]. Figure 4 shows that for the parameters of the simulation coherence is completely destroyed, which results in a monotonic behavior of efficiency and an essential increase in the maximum power. 

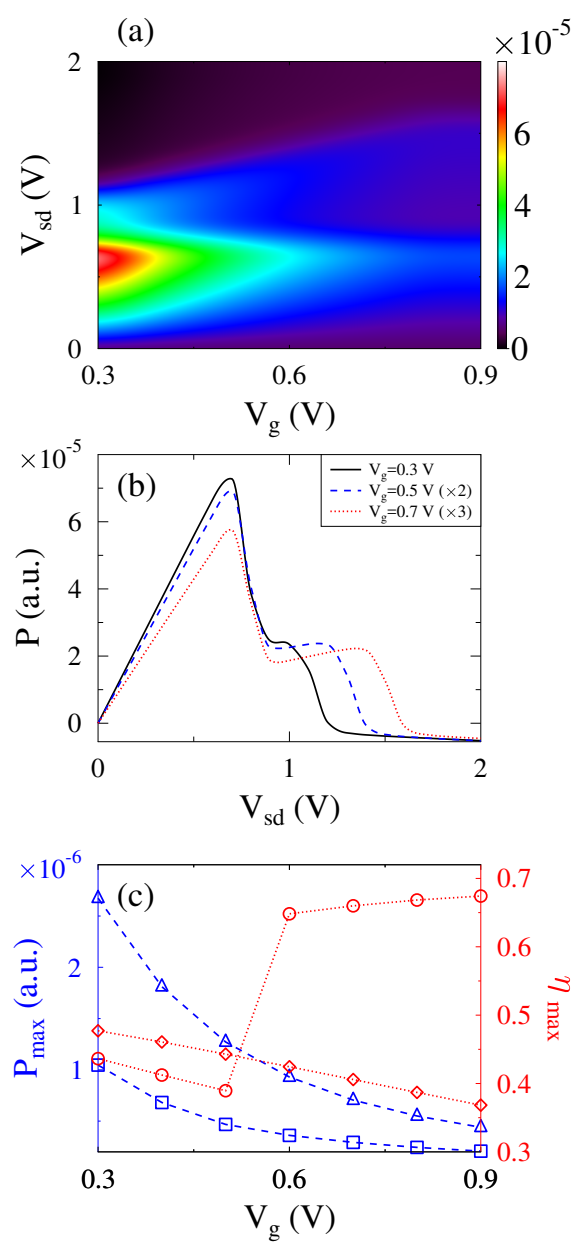

Figure 4. Coherence destruction on efficiency of the DBA heat engine by dissipation due to coupling to contacts. Shown are (a) a map of the engine power $P$ vs. detuning of the LUMOs, $V_{g}$, and bias across the junction, $V_{s d} ;(\mathbf{b})$ power $P$ vs. bias $V_{s d}$ for the three gate potentials (see inset; the results for $V_{g}=0.5$ and $0.7 \mathrm{~V}$ are scaled by a factor of 2 and 3, respectively); (c) the dependence of the maximum power $P_{\max }$ (dashed line, triangles) and efficiency at maximum power $\eta_{\max }$ (dotted line, diamonds) on gate potential $V_{g}$. The results presented in Figure 2c—squares (dashed line) and circles (dotted line)—are given for comparison.

\section{Conclusions}

We study thermoelectric properties of a bi-chromophoric DBA junction model driven by solar radiation of the donor complex. In particular, utilizing NEGF formalism, we calculate the efficiency at the maximum power and elucidate the role of intra-molecular quantum coherence in the efficiency. Coherence in the junction is controlled by effective donor-acceptor coupling and by detuning of molecular levels at the two nodes of the molecular complex. We show that quantum coherence results in non-monotonic behavior of efficiency at maximum power vs. level detuning. The observed sudden jump indicates a switch between dominant scattering eigenchannel in our two-site model (see Figure 1). Although the non-monotonic behavior of the current-voltage characteristic in junctions is well-known, thus far nobody has discussed this effect with respect to efficiency.

We stress that this behavior is possible only due to the presence of quantum coherence in the molecular system and that when coherence is destroyed, efficiency at maximum power attains its monotonic dependence on level detuning in agreement with previously performed classical studies of the system. To show the quantum-classical transition, we destroy quantum coherence in the system by either employing a Büttiker probe or increasing molecule-contacts coupling. In both cases, destruction 
of coherence implies a transition from two transport channels to one transport channel and from quantum to classical consideration. We indicate that employing a Büttiker probe results in pure dephasing with transition from coherent transport to hopping, thus reducing the quantum model to the classical consideration of [42]. At the same time, increasing the strength of molecule-contacts coupling leads to dissipation and effectively eliminates donor LUMO by incorporating it into the right contact. This reduces our model to the classical consideration of [41]. Comparing the classical and quantum results, we find that quantum coherence may be advantageous or disadvantageous for the performance of a molecular thermoelectric device (interference effects may lead to an increase or decrease in the efficiency).

Acknowledgments: We thank Paul Brumer and Christian Van den Broeck for helpful comments. This material is based upon work supported by the National Science Foundation under CHE-1565939.

Author Contributions: Feng Chen and Yi Gao performed simulations and prepared figures; Michael Galperin wrote the paper. All authors have read and approved the final manuscript.

Conflicts of Interest: The authors declare no conflict of interest.

\section{Abbreviations}

The following abbreviations are used in this manuscript:

$\begin{array}{ll}\text { EOM } & \text { Equation-Of-Motion } \\ \text { DBA } & \text { Donor-Bridge-Acceptor } \\ \text { HOMO } & \text { Highest Occupied Molecular Orbital } \\ \text { LUMO } & \text { Lowest Unoccupied Molecular Orbital } \\ \text { NEGF } & \text { Nonequilibrium Green Function }\end{array}$

\section{Appendix A. Expressions for Self-Energies}

Expressions for self-energies Equation (5) can be derived following the standard diagrammatic perturbation theory formulated on the Keldysh contour $[60,73,74]$. The resulting expression for the electron self-energies due to coupling to contacts $L$ and $R$ is exact

$$
\sum_{m_{1} m_{2}}^{K}\left(\tau_{1}, \tau_{2}\right)=\sum_{k \in K} V_{m_{1} k} g_{k}\left(\tau_{1}, \tau_{2}\right) V_{k m_{2}} \quad(K=L, R)
$$

where

$$
g_{k}\left(\tau_{1}, \tau_{2}\right) \equiv-i\left\langle\mathcal{T}_{c} \hat{c}_{l}\left(\tau_{1}\right) \hat{c}_{k}^{\dagger}\left(\tau_{2}\right)\right\rangle
$$

is the Green function of the free electron in state $k$. For Equation (2), $V_{m k}=V_{\ell}$ for $m=1$ and $V_{m k}=V_{r}$ for $m=3$. At steady state and within the wide-band approximation, the projections of (A1) after performing the Fourier transform are

$$
\begin{aligned}
& \sum_{m_{1} m_{2}}^{K--}(E)=\Sigma_{m_{1} m_{2}}^{K++}(E)=i \Gamma_{m_{1} m_{2}}^{K}\left(f_{K}(E)-\frac{1}{2}\right) \\
& \sum_{m_{1} m_{2}}^{K-+}(E)=i \Gamma_{m_{1} m_{2}}^{K} f_{K}(E) \\
& \sum_{m_{1} m_{2}}^{K+-}(E)=-i \Gamma_{m_{1} m_{2}}^{K}\left(1-f_{K}(E)\right)
\end{aligned}
$$

where $K=L, R$, superscript $-(+)$ indicates the forward (backward) branches of the Keldysh contour, $f_{K}(E)$ is the Fermi-Dirac distribution, and

$$
\Gamma_{m_{1} m_{2}}^{K}(E) \equiv 2 \pi \sum_{k \in K} V_{m_{1} k} V_{k m_{2}} \delta\left(E-\varepsilon_{k}\right)
$$


is the dissipation matrix due to coupling to contacts. In the wide-band approximation, the matrix is energy-independent. For Equation (2), $\Gamma_{m_{1} m_{2}}^{L}=\delta_{m_{1}, m_{2}, 1} \Gamma_{L}$ and $\Gamma_{m_{1} m_{2}}^{R}=\delta_{m_{1}, m_{2}, 3} \Gamma_{R}$, where $\Gamma_{L}$ and $\Gamma_{R}$ are the electron escape rates into contacts $L$ and $R$, respectively. Note $\Sigma^{K<(>)} \equiv \Sigma^{K-+(+-)}$.

The expression for the self-energy due to coupling to the thermal bath is derived within the second order of the diagrammatic perturbation theory

$$
\Sigma_{m_{1} m_{2}}^{S}\left(\tau_{1}, \tau_{2}\right)= \begin{cases}i \Pi\left(\tau_{2}, \tau_{1}\right) G_{22}\left(\tau_{1}, \tau_{2}\right) & \text { for } m_{1}=m_{2}=1 \\ i \Pi\left(\tau_{1}, \tau_{2}\right) G_{11}\left(\tau_{1}, \tau_{2}\right) & \text { for } m_{1}=m_{2}=2 \\ 0 & \text { otherwise }\end{cases}
$$

where

$$
\Pi\left(\tau_{1}, \tau_{2}\right) \equiv \sum_{\alpha}\left|U_{\alpha}\right|^{2} F_{\alpha}\left(\tau_{1}, \tau_{2}\right)
$$

is the self-energy due to the coupling of the molecular excitations to the thermal bath. The projections after the Fourier transform is performed are

$$
\Sigma_{m_{1} m_{2}}^{S_{1} s_{2}}(E)=i \int_{0}^{+\infty} \frac{d \omega}{2 \pi} \begin{cases}\Pi^{s_{2} s_{1}}(\omega) G_{22}^{s_{1} s_{2}}(E+\omega) & \text { for } m_{1}=m_{2}=1 \\ \Pi^{S_{1} s_{2}}(\omega) G_{22}^{S_{1} S_{2}}(E-\omega) & \text { for } m_{1}=m_{2}=2 \\ 0 & \text { otherwise. }\end{cases}
$$

Here, $s_{1,2}=\{-,+\}$ and

$$
\begin{aligned}
& \Pi^{--}(E)=\Pi^{++}(E)=-i \gamma\left(N(E)+\frac{1}{2}\right) \\
& \Pi^{-+}(E)=-i \gamma N(E) \\
& \Pi^{+-}(E)=-i \gamma(1+N(E))
\end{aligned}
$$

$N(E)$ is the Bose-Einstein distribution, and

$$
\gamma(\omega) \equiv 2 \pi \sum_{\alpha}\left|U_{\alpha}\right|^{2} \delta\left(\omega-\omega_{\alpha}\right)
$$

is the energy dissipation rate.

\section{Appendix B. Derivation of Fluxes}

The expression for electron flux, Equation (10), is the celebrated Jauho, Wingreen and Meir formula [75] written for the steady-state situation.

Here, we will focus on the derivation of the photon energy flux Equation (11). We start from the definition of the flux as the rate of the change of energy in the thermal bath (the radiation field)

$$
J_{S}(t) \equiv-\frac{d}{d t} \sum_{\alpha} \omega_{\alpha}\left\langle\hat{a}_{\alpha}^{\dagger}(t) \hat{a}_{\alpha}(t)\right\rangle
$$

Here, $\langle\ldots\rangle$ is the statistical and quantum mechanical averaging with the density operator of the whole world (system plus baths), and the operators are represented in the Heisenberg picture. Using the Heisenberg equation-of-motion (EOM) for the operators $\hat{a}_{\alpha}^{\dagger}(t)$ and $\hat{a}_{\alpha}(t)$ within Equation (2), one arrives at the expression for the flux

$$
J_{S}(t)=2 \operatorname{Re} \sum_{\alpha} \omega_{\alpha} U_{\alpha} G_{\alpha D}^{<}(t, t)
$$


in terms of the lesser projection of a mixed (photon-molecular excitation) Green function

$$
G_{\alpha D}\left(\tau_{1}, \tau_{2}\right) \equiv-i\left\langle\mathcal{T}_{c} \hat{a}_{\alpha}\left(\tau_{1}\right) \hat{D}^{\dagger}\left(\tau_{2}\right)\right\rangle
$$

Writing the Dyson equation for the Green function in the integral form and taking a lesser projection leads to

$$
G_{\alpha D}^{<}(t, t)=\int_{-\infty}^{+\infty} d t_{1}\left(F_{\alpha}^{<}\left(t, t_{1}\right) U_{\alpha}^{*} \mathcal{G}^{a}\left(t_{1}, t\right)+F_{\alpha}^{r}\left(t, t_{1}\right) U_{\alpha}^{*} \mathcal{G}^{<}\left(t_{1}, t\right)\right)
$$

where $\mathcal{G}$ is the two-particle Green function defined in Equation (3) and

$$
F_{\alpha}\left(\tau_{1}, \tau_{2}\right) \equiv-i\left\langle\mathcal{T}_{c} \hat{a}_{\alpha}\left(\tau_{1}\right) \hat{a}_{\alpha}^{\dagger}\left(\tau_{2}\right)\right\rangle
$$

is the Green function of the free photon of the radiation field. Substituting Equation (A17) into Equation (A15) and using

$$
\begin{gathered}
F_{\alpha}^{r}\left(t, t_{1}\right) \equiv \theta\left(t-t_{1}\right)\left(F_{\alpha}^{>}\left(t, t_{1}\right)-F_{\alpha}^{<}\left(t, t_{1}\right)\right) \\
\mathcal{G}^{a}\left(t_{1}, t\right) \equiv \theta\left(t-t_{1}\right)\left(\mathcal{G}^{<}\left(t_{1}, t\right)-\mathcal{G}^{>}\left(t_{1}, t\right)\right)
\end{gathered}
$$

(here, $\theta(x)$ is the Heaviside step function) leads to

$$
J_{S}(t)=-2 \operatorname{Re} \sum_{\alpha}\left|U_{\alpha}\right|^{2} \omega_{\alpha} \int_{-\infty}^{t} d t_{1}\left(\partial_{t} F_{\alpha}^{<}\left(t, t_{1}\right) \mathcal{G}^{>}\left(t_{1}, t\right)+\partial_{t} F_{\alpha}^{>}\left(t, t_{1}\right) \mathcal{G}^{<}\left(t_{1}, t\right)\right)
$$

Finally, taking into account that for the free photon in mode $\alpha F_{\alpha}^{<(>)}\left(t, t_{1}\right) \sim e^{-i \omega_{\alpha}\left(t-t_{1}\right)}$, and thus, $\omega_{\alpha} F_{\alpha}^{<(>)}\left(t, t_{1}\right) \equiv i \partial_{t} F_{\alpha}^{<(>)}\left(t, t_{1}\right)$, we get

$$
J_{S}(t)=-2 i \operatorname{Re} \int_{-\infty}^{t} d t_{1}\left(\partial_{t} \Pi^{<}\left(t, t_{1}\right) \mathcal{G}^{>}\left(t_{1}, t\right)-\partial_{t} \Pi^{>}\left(t, t_{1}\right) \mathcal{G}^{<}\left(t_{1}, t\right)\right)
$$

where $\Pi\left(\tau_{1}, \tau_{2}\right)$ is defined in Equation (A8). At steady-state, the correlation functions depend only on the time difference; therefore, performing the Fourier transform in Equation (A22) leads to Equation (11).

\section{References}

1. Aviram, A.; Ratner, M.A. Molecular Rectifiers. Chem. Phys. Lett. 1974, 29, 277-283.

2. Ratner, M. A Brief History of Molecular Electronics. Nat. Nanotech. 2013, 8, 378-381.

3. Van der Molen, S.J.; Naaman, R.; Scheer, E.; Neaton, J.B.; Nitzan, A.; Natelson, D.; Tao, N.J.; van der Zant, H.S.J.; Mayor, M.; et al. Visions for a Molecular Future. Nat. Nanotech. 2013, 8, 385-389.

4. Hensley, C.J.; Yang, J.; Centurion, M. Imaging of Isolated Molecules with Ultrafast Electron Pulses. Phys. Rev. Lett. 2012, 109, 133202.

5. Ham, U.; Ho, W. Imaging Single Electron Spin in a Molecule Trapped within a Nanocavity of Tunable Dimension. J. Chem. Phys. 2013, 138, 074703.

6. Schaffert, J.; Cottin, M.C.; Sonntag, A.; Karacuban, H.; Bobisch, C.A.; Lorente, N.; Gauyacq, J.P.; Möller, R. Imaging the Dynamics of Individually Adsorbed Molecules. Nat. Mater. 2013, 12, 223-227.

7. Zhang, R.; Zhang, Y.; Dong, Z.C.; Jiang, S.; Zhang, C.; Chen, L.G.; Zhang, L.; Liao, Y.; Aizpurua, J.; Luo, Y.; Yang, J.L.; Hou, J.G. Chemical Mapping of a Single Molecule by Plasmon-Enhanced Raman Scattering. Nature 2013, 498, 82-86.

8. Chiang, C.l.; Xu, C.; Han, Z.; Ho, W. Real-Space Imaging of Molecular Structure and Chemical Bonding by Single-Molecule Inelastic Tunneling Probe. Science 2014, 344, 885-888. 
9. Schwarz, F.; Kastlunger, G.; Lissel, F.; Egler-Lucas, C.; Semenov, S.N.; Venkatesan, K.; Berke, H.; Stadler, R.; Lörtscher, E. Field-Induced Conductance Switching by Charge-State Alternation in Organometallic Single-Molecule Junctions. Nat. Nanotech. 2016, 11, 170-176.

10. Mayor, M.; Weber, H.B.; Reichert, J.; Elbing, M.;von Hänisch, C.; Beckmann, D.; Fischer, M. Electric Current through a Molecular Rod-Relevance of the Position of the Anchor Groups. Angew. Chem. Int. Ed. 2003, $42,5834-5838$.

11. Vazquez, H.; Skouta, R.; Schneebeli, S.; Kamenetska, M.; Breslow, R.; Venkataraman, L.; Hybertsen, M. Probing the Conductance Superposition Law in Single-Molecule Circuits with Parallel Paths. Nature Nanotech. 2012, 7, 663-667.

12. Ballmann, S.; Härtle, R.; Coto, P.B.; Elbing, M.; Mayor, M.; Bryce, M.R.; Thoss, M.; Weber, H.B. Experimental Evidence for Quantum Interference and Vibrationally Induced Decoherence in Single-Molecule Junctions. Phys. Rev. Lett. 2012, 109, 056801.

13. Frontiera, R.R.; Gruenke, N.L.; Duyne, R.P.V. Fano-Like Resonances Arising from Long-Lived Molecule-Plasmon Interactions in Colloidal Nanoantennas. Nano Lett. 2012, 12, 5989-5994.

14. Lee, J.; Perdue, S.M.; Perez, A.R.; Apkarian, V.A. Vibronic Motion with Joint Angstrom-Femtosecond Resolution Observed through Fano Progressions Recorded within One Molecule. ACS Nano 2014, 8, 54-63.

15. Lee, H.; Cheng, Y.C.; Fleming, G.R. Coherence Dynamics in Photosynthesis: Protein Protection of Excitonic Coherence. Science 2007, 316, 1462-1465.

16. Engel, G.S.; Calhoun, T.R.; Read, E.L.; Ahn, T.K.; Mancal, T.; Cheng, Y.C.; Blankenship, R.E.; Fleming, G.R. Evidence for Wavelike Energy Transfer through Quantum Coherence in Photosynthetic Systems. Nature 2007, 446, 782-786.

17. Panitchayangkoon, G.; Hayes, D.; Fransted, K.A.; Caram, J.R.; Harel, E.; Wen, J.; Blankenship, R.E.; Engel, G.S. Long-Lived Quantum Coherence in Photosynthetic Complexes at Physiological Temperature. Proc. Natl. Acad. Sci. USA 2010, 107, 12766-12770.

18. Ishizaki, A.; Calhoun, T.R.; Schlau-Cohen, G.S.; Fleming, G.R. Quantum Coherence and its Interplay with Protein Environments in Photosynthetic Electronic Energy Transfer. Phys. Chem. Chem. Phys. 2010, 12, 7319-7337.

19. Ioffe, A.F. Semiconductor Thermoelements and Thermoelectric Cooling; Infosearch Ltd.: London, UK, 1957.

20. Kim, P.; Shi, L.; Majumdar, A.; McEuen, P.L. Thermal Transport Measurements of Individual Multiwalled Nanotubes. Phys. Rev. Lett. 2001, 87, 215502.

21. Reddy, P.; Jang, S.Y.; Segalman, R.A.; Majumdar, A. Thermoelectricity in Molecular Junctions. Science 2007, 315, 1568-1571.

22. Malen, J.A.; Yee, S.K.; Majumdar, A.; Segalman, R.A. Fundamentals of Energy Transport, Energy Conversion, and Thermal Properties in Organic-Inorganic Heterojunctions. Chem. Phys. Lett. 2010, 491, 109-122.

23. Widawsky, J.R.; Darancet, P.; Neaton, J.B.; Venkataraman, L. Simultaneous Determination of Conductance and Thermopower of Single Molecule Junctions. Nano Lett. 2012, 12, 354-358.

24. Tsutsui, M.; Morikawa, T.; Arima, A.; Taniguchi, M. Thermoelectricity in Atom-Sized Junctions at Room Temperatures. Sci. Rep. 2013, 3, 3326.

25. Brantut, J.P.; Grenier, C.; Meineke, J.; Stadler, D.; Krinner, S.; Kollath, C.; Esslinger, T.; Georges, A. A Thermoelectric Heat Engine with Ultracold Atoms. Science 2013, 342, 713-715.

26. Chabinyc, M. Thermoelectric Polymers: Behind Organics' Thermopower. Nature Mater. 2014, 13, $119-121$.

27. Kim, Y.; Jeong, W.; Kim, K.; Lee, W.; Reddy, P. Electrostatic Control of Thermoelectricity in Molecular Junctions. Nature Nanotech. 2014, 9, 881-885.

28. Lee, E.S.; Cho, S.; Lyeo, H.K.; Kim, Y.H. Seebeck Effect at the Atomic Scale. Phys. Rev. Lett. 2014, 112, 136601.

29. Paulsson, M.; Datta, S. Thermoelectric Effect in Molecular Electronics. Phys. Rev. B 2003, 67, 241403.

30. Humphrey, T.E.; Linke, H. Reversible Thermoelectric Nanomaterials. Phys. Rev. Lett. 2005, 94, 096601.

31. Galperin, M.; Nitzan, A.; Ratner, M.A. Inelastic Effects in Molecular Junction Transport: Scattering and Self-Consistent Calculations for the Seebeck Coefficient. Mol. Phys. 2008, 106, 397-404.

32. Liu, Y.S.; Chen, Y.R.; Chen, Y.C. Thermoelectric Efficiency in Nanojunctions: A Comparison between Atomic Junctions and Molecular Junctions. ACS Nano 2009, 3, 3497-3504. 
33. Liu, Y.S.; Chen, Y.C. Seebeck Coefficient of Thermoelectric Molecular Junctions: First-Principles Calculations. Phys. Rev. B 2009, 79, 193101.

34. Dubi, Y.; Di Ventra, M. Thermoelectric Effects in Nanoscale Junctions. Nano Lett. 2009, 9, 97-101.

35. Leijnse, M.; Wegewijs, M.R.; Flensberg, K. Nonlinear Thermoelectric Properties of Molecular Junctions with Vibrational Coupling. Phys. Rev. B 2010, 82, 045412.

36. Fransson, J.; Galperin, M. Spin Seebeck Coefficient of a Molecular Spin Pump. Phys. Chem. Chem. Phys. 2011, 13, 14350-14357.

37. Entin-Wohlman, O.; Imry, Y.; Aharony, A. Three-Terminal Thermoelectric Transport through a Molecular Junction. Phys. Rev. B 2010, 82, 115314.

38. Entin-Wohlman, O.; Aharony, A. Three-Terminal Thermoelectric Transport under Broken Time-Reversal Symmetry. Phys. Rev. B 2012, 85, 085401.

39. Nikolić, B.K.; Saha, K.K.; Markussen, T.; Thygesen, K.S. First-Principles Quantum Transport Modeling of Thermoelectricity in Single-Molecule Nanojunctions with Graphene Nanoribbon Electrodes. J. Comput. Electron. 2012, 11, 78-92.

40. Sánchez, D.; López, R. Scattering Theory of Nonlinear Thermoelectric Transport. Phys. Rev. Lett. 2013, $110,026804$.

41. Rutten, B.; Esposito, M.; Cleuren, B. Reaching Optimal Efficiencies Using Nanosized Photoelectric Devices. Phys. Rev. B 2009, 80, 235122.

42. Einax, M.; Dierl, M.; Nitzan, A. Heterojunction Organic Photovoltaic Cells as Molecular Heat Engines: A Simple Model for the Performance Analysis. J. Phys. Chem. C 2011, 115, 21396-21401.

43. Creatore, C.; Parker, M.A.; Emmott, S.; Chin, A.W. Efficient Biologically Inspired Photocell Enhanced by Delocalized Quantum States. Phys. Rev. Lett. 2013, 111, 253601.

44. Einax, M.; Nitzan, A. Network Analysis of Photovoltaic Energy Conversion. J. Phys. Chem. C 2014, 118, 27226-27234.

45. Einax, M.; Nitzan, A. Maximum Efficiency of State-Space Models of Molecular Scale Engines. arXiv 2015, arXiv:1506.00496.

46. Rahav, S.; Harbola, U.; Mukamel, S. Heat Fluctuations and Coherences in a Quantum Heat Engine. Phys. Rev. A 2012, 86, 043843.

47. Dorfman, K.E.; Voronine, D.V.; Mukamel, S.; Scully, M.O. Photosynthetic reaction center as a quantum heat engine. Proc. Natl. Acad. Sci. USA 2013, 110, 2746-2751.

48. Goswami, H.P.; Harbola, U. Thermodynamics of Quantum Heat Engines. Phys. Rev. A 2013, 88, 013842.

49. Altintas, F.; Hardal, A.U.C.; Müstecaplioğlu, O.E. Rabi Model as a Quantum Coherent Heat Engine: From Quantum Biology to Superconducting Circuits. Phys. Rev. A 2015, 91, 023816.

50. Leggio, B.; Bellomo, B.; Antezza, M. Quantum Thermal Machines with Single Nonequilibrium Environments. Phys. Rev. A 2015, 91, 012117.

51. Leijnse, M.; Wegewijs, M.R. Kinetic equations for transport through single-molecule transistors. Phys. Rev. B 2008, 78, 235424.

52. Esposito, M.; Galperin, M. Self-Consistent Quantum Master Equation Approach to Molecular Transport. J. Phys. Chem. C 2010, 114, 20362-20369.

53. Esposito, M.; Ochoa, M.A.; Galperin, M. Efficiency fluctuations in quantum thermoelectric devices. Phys. Rev. B 2015, 91, 115417.

54. Gao, Y.; Galperin, M. Simulation of optical response functions in molecular junctions. J. Chem. Phys. 2016, $144,244106$.

55. Kosloff, R.; Levy, A. Quantum Heat Engines and Refrigerators: Continuous Devices. Ann. Rev. Phys. Chem. 2014, 65, 365-393.

56. Proesmans, K.; Cleuren, B.; den Broeck, C.V. Stochastic efficiency for effusion as a thermal engine. Europhys. Lett. 2015, 109, 20004.

57. Kosloff, R.; Rezek, Y. The Quantum Harmonic Otto Cycle. Entropy 2017, 19, 136.

58. Peskin, U.; Galperin, M. Coherently Controlled Molecular Junctions. J. Chem. Phys. 2012, 136, 044107.

59. White, A.J.; Peskin, U.; Galperin, M. Coherence in Charge and Energy Transfer in Molecular Junctions. Phys. Rev. B 2013, 88, 205424.

60. Haug, H.; Jauho, A.P. Quantum Kinetics in Transport and Optics of Semiconductors; Springer: Berlin/Heidelberg, Germany, 2008. 
61. Aoki, H.; Tsuji, N.; Eckstein, M.; Kollar, M.; Oka, T.; Werner, P. Nonequilibrium Dynamical Mean-Field Theory and Its Applications. Rev. Mod. Phys. 2014, 86, 779-837.

62. White, A.J.; Ochoa, M.A.; Galperin, M. Nonequilibrium Atomic Limit for Transport and Optical Response of Molecular Junctions. J. Phys. Chem. C 2014, 118, 11159-11173.

63. Chen, F.; Ochoa, M.A.; Galperin, M. Nonequilibrium diagrammatic technique for Hubbard Green functions. J. Chem. Phys. 2017, 146, 092301.

64. Galperin, M. Photonics and spectroscopy in nanojunctions: A theoretical insight. Chem. Soc. Rev. 2017, 46, 4000-4019.

65. Miwa, K.; Chen, F.; Galperin, M. Towards Noise Simulation in Interacting Nonequilibrium Systems Strongly Coupled to Baths. Sci. Rep. 2017, 7, 9735.

66. Golizadeh-Mojarad, R.; Datta, S. Nonequilibrium Green's function based models for dephasing in quantum transport. Phys. Rev. B 2007, 75, 081301.

67. Esposito, M.; Ochoa, M.A.; Galperin, M. Nature of heat in strongly coupled open quantum systems. Phys. Rev. B 2015, 92, 235440.

68. Ochoa, M.A.; Bruch, A.; Nitzan, A. Energy distribution and local fluctuations in strongly coupled open quantum systems: The extended resonant level model. Phys. Rev. B 2016, 94, 035420.

69. Gaspard, P. Scattering approach to the thermodynamics of quantum transport. New J. Phys. 2015, $17,045001$.

70. Ness, H. Nonequilibrium Thermodynamics and Steady State Density Matrix for Quantum Open Systems. Entropy 2017, 19, 158.

71. Galperin, M.; Nitzan, A. Optical Properties of Current Carrying Molecular Wires. J. Chem. Phys. 2006, $124,234709$.

72. Scully, M.O. Quantum Photocell: Using Quantum Coherence to Reduce Radiative Recombination and Increase Efficiency. Phys. Rev. Lett. 2010, 104, 207701.

73. Fetter, A.L.; Walecka, J.D. Quantum Theory of Many-Particle Systems; Dover Publications, Inc.: Mineola, NY, USA, 2003.

74. Stefanucci, G.; van Leeuwen, R. Nonequilibrium Many-Body Theory of Quantum Systems. A Modern Introduction; Cambridge University Press: Cambridge, UK, 2013.

75. Jauho, A.P.; Wingreen, N.S.; Meir, Y. Time-dependent transport in interacting and noninteracting resonant-tunneling systems. Phys. Rev. B 1994, 50, 5528-5544.

(C) 2017 by the authors. Licensee MDPI, Basel, Switzerland. This article is an open access article distributed under the terms and conditions of the Creative Commons Attribution (CC BY) license (http:/ / creativecommons.org/licenses/by/4.0/). 